\title{
Les profits «excessifs 》 des commerçants de fruits et légumes en Algérie : réalité ou préjugé infondé ? Le cas de la tomate primeur à Biskra
}

\author{
Sami Assassi ${ }^{1, *}$, Ali Daoudi ${ }^{1}$ et Caroline Lejars ${ }^{2}$ \\ ${ }^{1}$ École nationale supérieure agronomique (ENSA), avenue Hassan Badi, El Harrach, Algérie \\ ${ }^{2}$ Cirad, UMR G-Eau, 34398 Montpellier cedex 5, France
}

\begin{abstract}
Résumé - Dans les pays en développement, les agriculteurs dépendent, pour la commercialisation de leurs produits, d'un réseau complexe d'intermédiaires, souvent accusés de réaliser des profits excessifs. C'est le cas en Algérie, où ce discours est relayé par les agriculteurs et les consommateurs. Est-ce une réalité ou un préjugé infondé ? Cette étude se focalise sur la filière tomate primeur d'El Ghrous (wilaya de Biskra, dans le Sahara algérien), dont la production a connu un développement fulgurant ces 20 dernières années. Dans un premier temps, les facteurs, endogènes et exogènes, déterminant la formation des prix sont identifiés. Dans un second temps, la répartition de la valeur ajoutée le long de la filière est analysée à travers le calcul du délai de récupération des fonds investis et une comparaison des taux de marge des acteurs. Les résultats montrent que, pour cette filière, les intermédiaires ne réalisent pas de profits excessifs au détriment des agriculteurs. Le marché de gros d'El Ghrous est efficace, ce qui constitue un atout majeur pour le développement des cultures maraîchères dans la région.
\end{abstract}

Mots clés : valeur ajoutée / circuit de commercialisation / filière / tomate / vente en gros / Algérie

\begin{abstract}
Excessive" profits of fruit and vegetables traders in Algeria: a reality or an unfounded prejudice? The case of early tomato from Biskra. In developing countries, farmers depend on a complex network of private intermediaries to sell their products. Hence, these intermediates are often accused of making excessive profits. It is the case in Algeria, where farmers and consumers spread this message. Is it a reality or an unfounded prejudice? To answer this question, this study focuses on the early tomato sector in the region of El Ghrous (Biskra, in Algerian Sahara). First, the endogenous and exogenous factors determining the price formation are identified. Second, the distribution of added value along the chain is analyzed. Through the calculation of payback period and the comparison of participants' margin rates, we show that intermediates do not make profits at farmers' expense and that the El Ghrous wholesale market is efficient.
\end{abstract}

Keywords: added value / marketing channels / value chain / tomato / wholesale marketing / Algeria

\section{Introduction}

$\mathrm{Au}$ Maghreb, comme dans beaucoup de régions en voie de développement, les agriculteurs dépendent souvent, pour la commercialisation de leurs produits, de réseaux complexes d'intermédiaires privés. L'effet de ces intermédiaires sur la compétitivité des filières fait l'objet de débats. Si le plus souvent l'importance de leur rôle comme catalyseurs de compétitivité des filières est soulignée (Gouyon, 1995), ils restent soupçonnés d'avoir des comportements opportunistes

\footnotetext{
* Auteur de correspondance : assassisami@hotmail.com
}

et de réaliser des profits excessifs au détriment des agriculteurs ou des consommateurs. C'est particulièrement le cas dans des contextes où les marchés sont opaques (Galtier, 2002 ; David-Benz et al., 2005), lorsque les informations concernant les caractéristiques des produits et leurs prix peuvent être dissimulées (Boussard, 2010). Dans des contextes où ces intermédiaires sont peu nombreux, ils peuvent également s'arranger pour empêcher l'émergence de concurrents et s'entendre pour limiter les prix payés aux agriculteurs (Lothoré et Delmas, 2009). Sur des filières de produits non périssables, le stockage leur donne parfois la capacité de fausser le jeu offre-demande et de spéculer « sur le dos » des agriculteurs et des consommateurs (Lejars et Courilleau, 2015). 
L'Algérie ne fait pas exception : un tel discours négatif sur les intermédiaires y est souvent relayé par les agriculteurs, les consommateurs et les institutions chargées de la régulation des marchés agricoles. Faute de transparence dans le fonctionnement de ces marchés, les intermédiaires sont considérés comme des spéculateurs qui manipulent les prix et entravent la bonne répartition des gains entre les acteurs de la filière (Réflexion, 2015). Dans le cas des produits maraîchers, la libéralisation des marchés entamée en 1987 a surtout bénéficié aux acteurs les mieux informés, tels que les mandataires des halles (Guillermou, 1994). Dans plusieurs cas, les longs circuits de distribution et l'opacité des marchés profitent surtout aux intermédiaires rentiers, n'ayant aucune relation avec l'agriculture et opérant aux différents stades des filières (Baci, 1995). D'autres études plus récentes, sur le marché de la viande rouge en Algérie, confirment leur rôle spéculatif dans les longs circuits de distribution. Étant les acteurs les mieux informés sur les prix nationaux, les maquignons «spéculateurs » profitent du manque de transparence pour réaliser rapidement des profits excessifs (près de deux fois plus que les profits des éleveurs) sans aucune transformation du produit (Zoubeidi et Chehat, 2011). Toutefois, le partage de la valeur ajoutée reste fortement dépendant du comportement des intermédiaires auxquels sont liés les agriculteurs (Sahli, 2009). Ces différences s'expliqueraient, en partie, par les spécificités des produits agricoles eux-mêmes et la structure de leurs circuits de commercialisation.

La présente étude se focalise sur le cas de la filière tomate fraîche primeur et le marché de gros d'El Ghrous, dans la wilaya (département) de Biskra. La production de tomate dans cette région saharienne a connu un développement extraordinaire ces 20 dernières années, positionnant la région en pôle de production de tomate primeur du pays (Daoudi et Lejars, 2016). Le marché d'El Ghrous est ainsi devenu, en quelques années, un des plus grands de la région. La tomate primeur, produit frais de large consommation, est commercialisée à travers un circuit court qui fait intervenir un petit nombre d'intermédiaires et dont la logistique est rudimentaire (emballage en plastique, transport en camions bâchés non appropriés, absence d'entrepôts à température contrôlée), ce qui réduit considérablement la durée de vie du produit.

L'objectif de l'étude est d'analyser les facteurs expliquant la formation des prix et les pratiques des intermédiaires de commercialisation, puis d'évaluer les marges des différents acteurs de la filière. L'hypothèse retenue est que, contrairement aux discours négatifs sur les intermédiaires des marchés de fruits et légumes, leurs pratiques et l'efficacité de ce marché en expansion sont une des clés du développement de la filière tomate primeur dans la région.

Dans un premier temps, la zone de production et le marché étudiés sont présentés, ainsi que le cadre d'analyse et les enquêtes effectuées. Les résultats sont présentés ensuite en deux sous-parties: l'analyse des facteurs endogènes et exogènes déterminant la formation des prix, puis l'évaluation des marges des acteurs de la filière à travers le calcul du délai de récupération des fonds investis et des taux de marge des acteurs. Enfin, dans la dernière partie, nous discutons le rôle clé de ce marché dans le développement local.

\section{Cas d'étude et méthodologie}

\subsection{La filière tomate primeur dans la région de Biskra}

Biskra, le site d'étude, est la principale zone de production de tomate en Algérie. Depuis longtemps, la wilaya occupait le premier rang national dans la production de dattes. Parallèlement à la filière dattes, le maraîchage sous serre, introduit en 1984 dans la région, a connu un développement fulgurant. Entre 1998 et 2013, la part de la production nationale sous serre provenant de Biskra a augmenté de près de $40 \%$. La superficie qu'occupe la «plasticulture » (le maraîchage sous serre plastique) à Biskra est passée de 1370 à 4050 ha entre 1999 et 2013 (DSA, 2013). Occupant actuellement moins de $1 \%$ de la superficie agricole totale et moins de $4 \%$ des superficies irriguées de la wilaya, la " plasticulture » contribue à plus de $50 \%$ à la production nationale sous serre (MADR, 2013).

La production maraîchère est concentrée principalement dans les communes d'El Ghrous et de M'Ziraâ, qui assurent respectivement 12 et $22 \%$ de la production de la wilaya (Fig. 1).

À Biskra, la tomate est produite principalement sous serre (cas de la tomate primeur d'El Ghrous) et c'est la culture maraîchère la plus importante. Elle occupe près de $45 \%$ des superficies couvertes par les serres (DSA, 2013). Elle est cultivée en utilisant principalement deux variétés hybrides appelées localement Tofane et Sahra.

L'avantage comparatif de la tomate primeur provient principalement des conditions climatiques de Biskra (région saharienne, ensoleillée et avec des températures relativement élevées) ainsi que de l'accès des agriculteurs aux eaux souterraines. Dans ces conditions climatiques, la tomate, qui est un produit périssable, manifeste les premiers signes de pourrissement 24 à $36 \mathrm{~h}$ après sa récolte. Destinée dans sa totalité au marché national, la tomate produite à Biskra est écoulée à travers des circuits courts, sans stockage (Fig. 2).

L'essentiel des quantités produites à Biskra transite par cinq marchés de gros, dont le plus important est celui d'El Ghrous, créé en 1992 pour faciliter l'écoulement des productions croissantes dans cette zone. Ce marché a écoulé plus de $40 \%$ de la production agricole de la wilaya en 2013. Il a engendré, durant la même année, des transactions d'un montant total estimé à près de 400 millions d'euros (MADR, 2013 ; DSA, 2013).

Ce marché est actuellement administré par cinq gestionnaires qui se chargent principalement de la sécurité et de l'encaissement des frais d'entrée des agriculteurs et grossistes. Les agriculteurs d'El Ghrous et des communes voisines (Doucen, Foughala, Lioua et Tolga) vendent leurs récoltes directement sur le marché aux grossistes qui viennent de presque tout le pays. Toutes les quantités échangées passent par les balances des agents de pesée, dont le rôle principal est de peser la marchandise une fois la transaction conclue. La principale spécificité de ce marché est l'absence de mandataires chargés de l'intermédiation entre les agriculteurs et les grossistes (c'est-à-dire de personnes chargées de la vente sur les marchés de gros, à qui les agriculteurs cèdent la marchandise et donc le pouvoir décisionnel de sa commercialisation, comme la négociation des prix ou le choix du moment de la vente et des clients). 


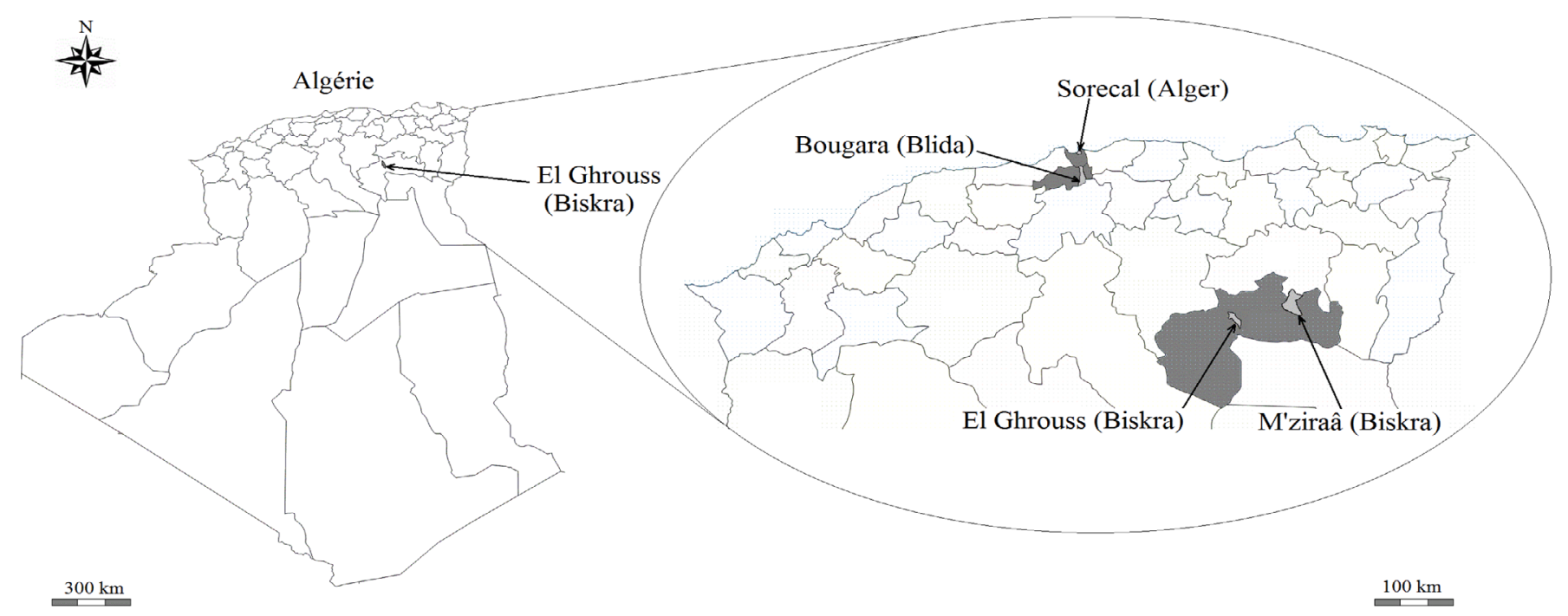

Fig. 1. Emplacement de la zone d'étude, El Ghrous.

Fig. 1. Location of the study area, El Ghrous.

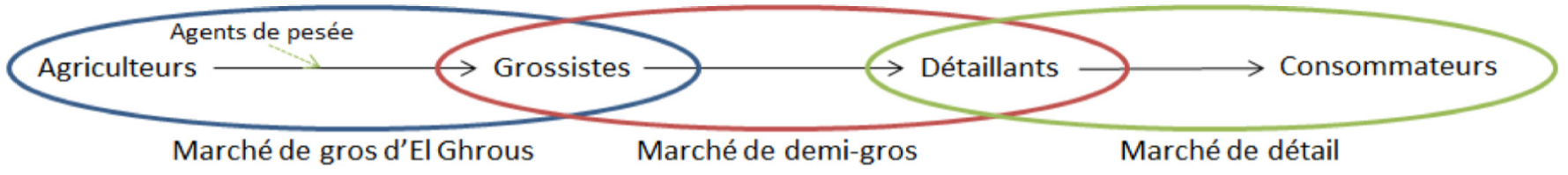

Fig. 2. Circuit de distribution de la tomate primeur.

Fig. 2. Early tomato distribution chain.

Les grossistes transportent les marchandises (le jour même, dans des camions non frigorifiés) vers les marchés de demigros du nord du pays, particulièrement ceux de Bougara (Blida) et d'Attatba (Tipaza), où ils les vendent aux détaillants chargés de la vente aux consommateurs finaux. Les détaillants assurent ensuite le transport des marchandises (immédiatement, dans des camionnettes) vers les marchés de détail des centres urbains (Fig. 2).

\subsection{Méthodologie}

\subsubsection{Le cadre d'analyse}

Le partage des marges bénéficiaires entre agriculteurs et intermédiaires (grossistes et détaillants) dépend de plusieurs facteurs, dont certains sont liés à la nature du produit commercialisé, à la structure de l'offre et de la demande et au fonctionnement des marchés. Afin de l'évaluer, nous déterminons dans un premier temps les facteurs expliquant la formation des prix sur le marché, en séparant les facteurs endogènes et exogènes. Nous apprécions dans un second temps le partage de la valeur ajoutée.

Les facteurs endogènes analysés concernent le système d'information du marché, notamment les modes d'accès aux informations déterminant les anticipations des acteurs (caractéristiques des produits et leurs prix) (Galtier et al., 2012). Les pratiques anticoncurrentielles des grossistes et détaillants, notamment en matière de transmission des variations des prix, sont ensuite identifiées. Les facteurs exogènes analysés concernent notamment les aléas climatiques qui influencent l'offre et la demande (Boussard, 2010). Leur effet sur la variation des prix est montré. Enfin, les bénéfices dégagés par les différents acteurs sont appréciés à travers un calcul du délai de récupération des fonds investis par les agriculteurs et une comparaison des taux de marge de tous les intervenants de la filière (Tarchiani et al., 2013). Ces deux méthodes se fixent respectivement comme objectif : l'évaluation de la rentabilité de la culture pour les agriculteurs et l'évaluation du partage de la valeur ajoutée.

Le délai de récupération représente le rapport des investissements totaux au bénéfice net d'une campagne : délai de récupération $=\frac{\text { investissements totaux }}{\text { bénéfice net d'une campagne }}$. Le taux de marge est une évaluation du bénéfice net d'un agent économique sur la base des coûts engagés pour la réalisation d'une activité quelconque : taux de marge $=\frac{\text { bénéfice net }}{\text { côtts totaux }} \times 100$.

\subsubsection{Les enquêtes}

La collecte des données a été effectuée durant toute la campagne 2012-2013, pour deux variétés de tomate primeur. Un suivi journalier de dix exploitations productrices de tomate (cinq cultivant la variété Tofane et cinq cultivant la variété Sahra, choisies à partir d'échantillonnage en boule de neige) a permis de calculer précisément les coûts de production au champ et d'obtenir une série de prix de vente par les agriculteurs. 


\begin{tabular}{|c|c|c|c|c|c|c|c|c|c|c|c|c|}
\hline Zonnes productrices & $\mathbf{J}$ & $\mathrm{F}$ & M & $\mathrm{A}$ & $\mathrm{M}$ & $\mathbf{J}$ & $\mathrm{J}$ & A & $\mathrm{S}$ & $\mathrm{O}$ & $\mathrm{N}$ & $\mathrm{D}$ \\
\hline \multicolumn{13}{|l|}{ Adrar } \\
\hline \multicolumn{13}{|l|}{ Biskra } \\
\hline \multicolumn{13}{|l|}{ Mostaganem } \\
\hline \multicolumn{13}{|l|}{ Tipaza } \\
\hline \multicolumn{13}{|l|}{ Jijel } \\
\hline Oued Souf & & & & & & & & & & & & \\
\hline
\end{tabular}

Fig. 3. Les différents calendriers de récolte des zones productrices de tomate fraîche en Algérie.

Fig. 3. Harvest calendars of fresh tomato producing areas in Algeria.

Sur le marché d'El Ghrous, cinq grossistes (choisis aléatoirement), six agents de pesée (tirés au hasard par strates), tous les gestionnaires du marché (cinq) ainsi que cinq détaillants s'approvisionnant sur le marché de Bougara (choisis aléatoirement) ont été suivis. Les enquêtes auprès des grossistes et détaillants ont permis de calculer leurs coûts (coûts de fonctionnement, quantités commercialisées et prix d'achat et de vente). Le suivi quotidien des agents de pesée et des gestionnaires du marché a permis d'estimer les quantités de tomate qui transitent par le marché et le nombre d'agriculteurs et de grossistes entrés quotidiennement. Les prix de gros ont été collectés lors de visites quotidiennes au marché. Ceux pris en considération représentent la moyenne des prix de vente prélevés, à la même heure, auprès des agriculteurs vendeurs de tomate de bonne qualité (rouge, calibrée et bien emballée).

Enfin, un relevé des prix a été fait par téléphone tout au long de la filière, depuis l'agriculteur jusqu'au marché d'Alger, pendant la période du 23 janvier au 6 février, sur le marché de gros d'El Ghrous, sur le marché de gros de Bougara (Blida), où vendent la majorité des grossistes interrogés, et sur le marché de détail d'un quartier populaire à Alger (marché Sorecal, de la cité 8 Mai 1945, commune de Bab-Ezzouar). Le choix de cette période relativement courte se justifie par le fait que pendant cet intervalle de temps, le marché national est exclusivement approvisionné en tomate provenant de Biskra, et particulièrement de la commune d'El Ghrous (Fig. 3). Cela garantit l'origine des signaux des prix transmis verticalement à travers les marchés choisis.

\section{Résultats}

\subsection{Les facteurs expliquant la formation des prix}

\subsubsection{Structuration du marché, système d'information et transparence}

Le système d'information officiel qui consiste à afficher les prix pratiqués la journée précédente, exigé par la réglementation du fonctionnement des marchés de gros (article 23 de l'arrêté interministériel du 6 février 1994 portant sur le règlement intérieur du service général des marchés de gros des fruits et légumes), est absent du marché d'El Ghrous. Même les outils de base, tels que l'éclairage et les moyens de pesée modernes, qui permettent de faciliter la récolte individuelle des informations, notamment celles relatives aux quantités et à la qualité des produits mis en vente, sont loin d'être le point fort du marché. D'un point de vue théorique, ces insuffisances entravent la bonne structuration des prix.

En moyenne quotidienne, le marché est fréquenté par 538 agriculteurs avec une capacité de vente individuelle moyenne de 1,6 t, et 120 grossistes avec une capacité d'achat individuelle moyenne de $8 \mathrm{t}$ (liée à la taille des camions). Le rapport de force entre ces deux acteurs est souvent en faveur des grossistes, car les agriculteurs sont contraints d'écouler rapidement leurs récoltes de produits périssables.

Les grossistes ne sont pas tous semblables. Certains ont de grandes capacités d'achat, estimées par le nombre et la capacité de leurs camions. À titre d'exemple, le 28 mars 2013, $23 \%$ des grossistes présents possédaient $50 \%$ des camions recensés sur le marché, soit deux à cinq camions par grossiste, d'une capacité de 4,5 à 8,5 t chacun. Les décisions d'achat (quantité et moment) de ces grands grossistes ont une forte influence sur les prix.

\subsubsection{La transmission des prix à travers la filière}

Les enquêtes réalisées montrent que la transmission des prix le long de la filière est globalement bonne (Fig. 4 et 5). Le produit peut arriver au marché de détail de Sorecal à Alger le jour même de sa commercialisation sur le marché d'El Ghrous, ou au plus tard le lendemain. Les changements de prix sur le marché d'El Ghrous (hausses et baisses) doivent normalement être transmis intégralement au marché de détail soit le jour même, soit avec un décalage d'une journée au maximum.

Comme le montrent ces figures, 18 fluctuations de prix sont notées sur le marché de gros pour les deux variétés pendant les 15 jours, neuf diminutions et neuf augmentations. Parmi ces dernières, sept ont été transmises directement aux autres marchés. Par contre, seulement trois parmi les neuf diminutions observées ont été transmises rapidement, quatre après une journée et deux n'ont jamais été transmises.

Ainsi, sur les 15 jours du suivi, une imperfection de la transmission des prix depuis le marché d'El Ghrous jusqu'au marché de détail est à signaler. Bien entendu, il est difficile de s'assurer d'une manière certaine de l'origine de ces changements. Néanmoins, la tendance des grossistes et des détaillants à transmettre plus rapidement aux consommateurs les hausses que les baisses des prix suggèrent que la transmission asymétrique des prix se fait plutôt en faveur des intermédiaires. 


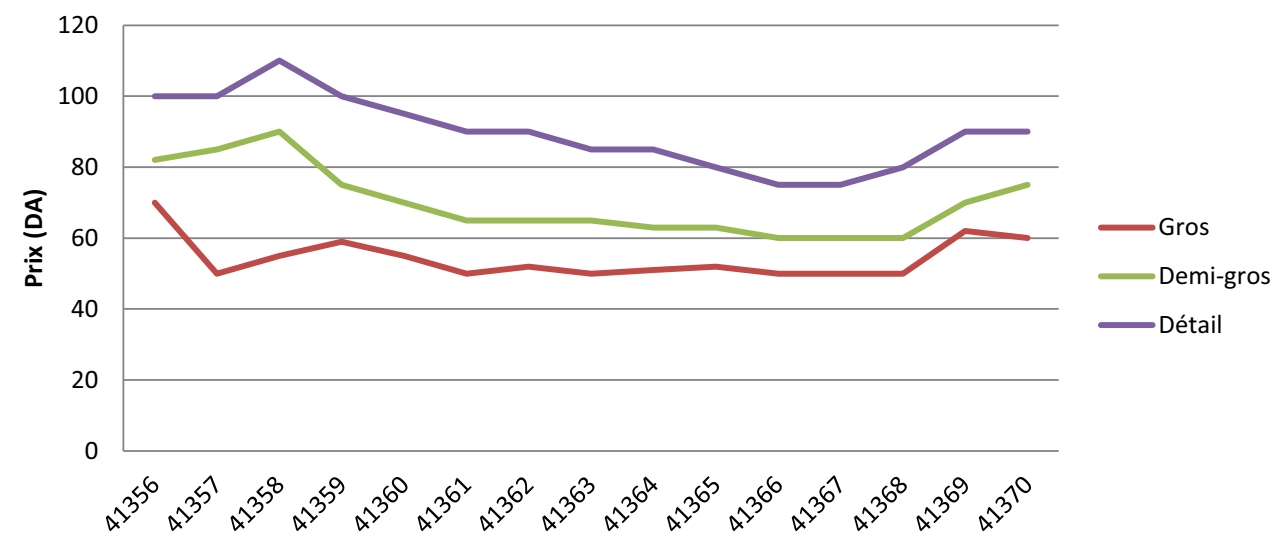

Fig. 4. Prix aux différentes étapes de la filière de la tomate, variété Tofane. $1 \mathrm{DA}=0,008$ euros.

Fig. 4. Prices at different steps of the tomato chain, Tofane variety.

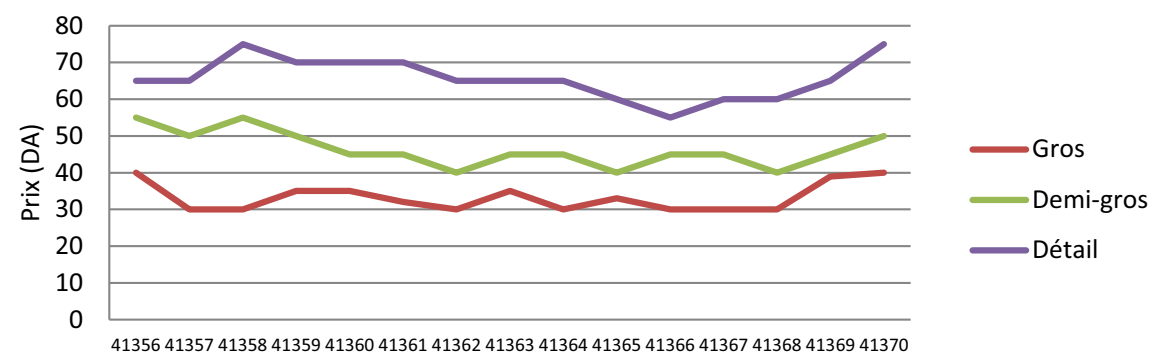

Fig. 5. Prix aux différentes étapes de la filière de la tomate, variété Sahra.

Fig. 5. Price at different steps of the tomato chain, Sahra variety.

\subsubsection{Les facteurs exogènes : l'effet du jeu de l'offre et de la demande sur les prix}

Les chocs exogènes liés aux aléas climatiques, naturels et phytopathologiques peuvent agir de différentes façons. Dans la zone d'étude, les fortes pluies et la neige génèrent principalement des empêchements routiers rendant difficile, voire impossible, le transport de la marchandise entre les zones de production(Biskra est à $440 \mathrm{~km}$ au Sud d'Alger) et les grands centres urbains du Nord du pays. Ces aléas pèsent différemment sur chaque segment de la filière. Ils diminuent la demande sur le marché de gros et, parallèlement, baissent l'offre sur le marché de demigros et de détail. Cela diminue le prix sur le premier et l'augmente sur les deux autres. Ces aléas peuvent aussi influencer les dates de récolte et le processus de mûrissement des fruits (tomate), et donc la production et, de ce fait, le niveau de l'offre et les prix.

Aux phénomènes cités, s'ajoute l'effet de l'entrée en production des zones productrices de tomate fraîche, sur l'offre nationale et sur les prix. La courbe tendancielle des prix de la tomate primeur nous renseigne sur la saisonnalité des quantités offertes et des prix correspondants (Fig. 6). L'effet des aléas climatiques, du décalage des calendriers et des pratiques des acteurs modifie ponctuellement ce facteur cyclique et donne à la courbe sa forme effective.

Comme le montre la Figure 6, les aléas climatiques enregistrés durant la campagne considérée et le décalage des calendriers de récolte influent fortement sur les prix en affectant l'offre. À titre d'exemple, la fin de la récolte dans la région
d'Oued Souf a diminué les quantités offertes sur le marché national et engendré une hausse des prix dès le 28 décembre.

L'effet de ces aléas est facilement observable, ce qui renforce l'idée que le prix de la tomate fraîche est le résultat de l'égalisation de l'offre et la demande. Les périodes du 30 décembre au 7 janvier, du 5 février au 7 février, ainsi que le 12 mars, ont été marquées par de basses températures $(12,11$ et $13^{\circ} \mathrm{C}$ ) par rapport à la moyenne enregistrée habituellement à ces périodes $\left(16^{\circ} \mathrm{C}\right)$. Cela a provoqué un ralentissement du mûrissement, donc une diminution des quantités récoltées, et, de ce fait, une augmentation des prix.

Les enquêtes ont montré que les périodes du 24 janvier au 25 janvier, du 27 février au 1 mars, ainsi que le 5 mars et le 6 mars, ont respectivement été marquées par les événements suivants qui ont induit une diminution des prix :

- chute importante de neige dans les régions Nord, ce qui a provoqué de grandes perturbations de la circulation routière et empêché l'arrivée des grossistes de certaines zones ;

- une augmentation de la température $\left(15^{\circ} \mathrm{C}\right)$, qui a accéléré le processus de mûrissement et, par conséquent, a augmenté les quantités offertes ;

- de fortes pluies dans les régions Nord, qui ont perturbé les déplacements des grossistes ( 80 grossistes ont fréquenté le marché ce jour au lieu de 120 habituellement).

Il y a lieu de souligner que l'effet de ces deux facteurs déterminants que sont l'offre et la demande est assez visible sur 


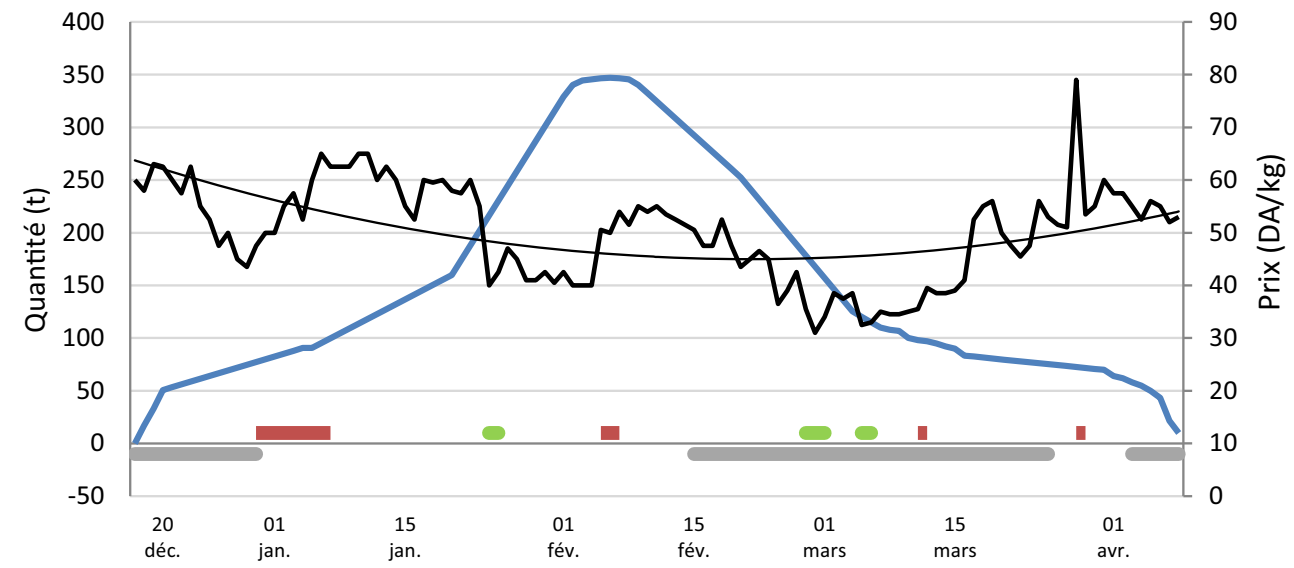

Quantité journalière de tomate primeur vendue sur le marché d'El Ghrous

Période marquée par des aléas climatiques susceptibles d'augmenter les prix

Période marquée par des aléas climatiques susceptibles de baisser les prix

Période où le marché national est alimenté en tomate provenant des autres régions du pays
Fig. 6. Prix de la tomate primeur et principaux facteurs exogènes les affectant.

Fig. 6. Early tomato prices and major exogenous factors.

Tableau 1. Délai de récupération du capital investi pour une serre de tomate.

Table 1. Payback of the capital invested on a tomato greenhouse.

Produit brut (DA)

Valeur ajoutée brute (DA)

Amortissement (DA)

Valeur ajoutée annuelle nette (DA)

Investissement (DA)

Délai de récupération
Prix $\times$ quantité

Produit brut - consommation intermédiaire

Serre + terre + forage

Valeur ajoutée brute - amortissement

Consommation intermédiaire + achat outillage + achat terre + réalisation du forage

Investissement / valeur ajoutée annuelle nette
178000

121000

26000

95000

294000

3 ans

$1 \mathrm{DA}=0,008$ euros.

le niveau des prix. Ces facteurs n'expliquent pas la totalité des fluctuations remarquées. Plusieurs changements de niveau de prix repérés ne coïncident avec aucun aléa climatique, à savoir les baisses du 15 janvier et du 21 mars et les hausses du 18 février et du 24 mars. Il est clair que les pratiques des acteurs ont aussi une influence.

\section{2 Évaluation des marges bénéficiaires}

Le délai de récupération du capital investi, estimé pour une serre de tomate en prenant en considération les autres cultures réalisées pendant la même saison (melon et pastèque), est de trois ans en moyenne (Tab. 1), renseignant ainsi sur la bonne rémunération des agriculteurs dans cette activité « plasticole » à moyen terme.

Pour la comparaison des taux de marge (Tab. 2), les coûts et les bénéfices sont calculés sur la base des quantités réellement achetées et écoulées par chaque acteur et sont rapportés à $1 \mathrm{~kg}$ de tomate pour lequel toutes les dépenses sont prises en considération.

Les prix moyens du kilogramme de tomate, relevés pendant la période du suivi sur les trois marchés d'El Ghrous, Bougara et Sorecal, sont respectivement de 44,58 et $78 \mathrm{DA}$, soit respectivement $0,37,0,5$ et 0,67 euros. Cela correspond respectivement, pour chacun des participants (agriculteurs, grossistes et détaillants), à un bénéfice net moyen de 10, 12 et $17 \mathrm{DA}$.
La répartition des marges bénéficiaires entre les différents acteurs, pendant la période considérée, est assez équilibrée dans l'ensemble. Les légères variations journalières s'expliquent en grande partie par le jeu de l'offre et de la demande (Fig. 7).

\section{Discussion et conclusion}

La présente étude a permis d'évaluer la répartition des marges bénéficiaires le long de la filière de la tomate primeur (El Ghrous, Biskra). Les brusques fluctuations quotidiennes des prix, qui rendent peu visible la répartition des bénéfices à court terme, nous ont conduits à calculer le délai de récupération du capital investi et le taux de marge. Le premier, qui est de trois ans, montre la rentabilité de la production de tomate primeur pour les agriculteurs. Le deuxième indice montre que la répartition des marges est presque égale et que les intermédiaires ne réalisent pas de profits excessifs au détriment des agriculteurs. Cela remet en question l'hypothèse d'une asymétrie des rapports de négociation au détriment des agriculteurs.

L'étude a aussi mis en évidence que le marché d'El Ghrous est efficace, contrairement à ce que laissent croire les premières apparences liées à l'organisation défaillante (pas d'éclairage, pratique de tricherie sur les pesées). La bonne 
Tableau 2. Calcul des coûts totaux pour $1 \mathrm{~kg}$ de tomates, pour tous les participants de la filière tomate.

Table 2. Calculation of total costs for $1 \mathrm{~kg}$ of tomatoes, for all participants of the tomato chain.

\begin{tabular}{|c|c|c|c|c|c|}
\hline Acteurs & Type de dépense & $\begin{array}{l}\text { Coûts totaux } \\
\text { (DA) }\end{array}$ & $\begin{array}{l}\text { Prix de } \\
\text { vente (DA) }\end{array}$ & $\begin{array}{l}\text { Bénéfices } \\
\text { nets (DA) }\end{array}$ & $\begin{array}{l}\text { Taux de } \\
\text { marge }(\%)\end{array}$ \\
\hline Agriculteurs & $\begin{array}{l}\text { Frais de location de la terre, amortissement, } \\
\text { consommation intermédiaire, employés, transport, } \\
\text { frais d'accès au marché et autres services }\end{array}$ & 34 & 44 & 10 & 28 \\
\hline Grossistes & $\begin{array}{l}\text { Carburant, amortissement du camion, frais de } \\
\text { commercialisation, employés, prix d'achat }\end{array}$ & 46 & 58 & 12 & 26 \\
\hline
\end{tabular}

$1 \mathrm{DA}=0,008$ euros.

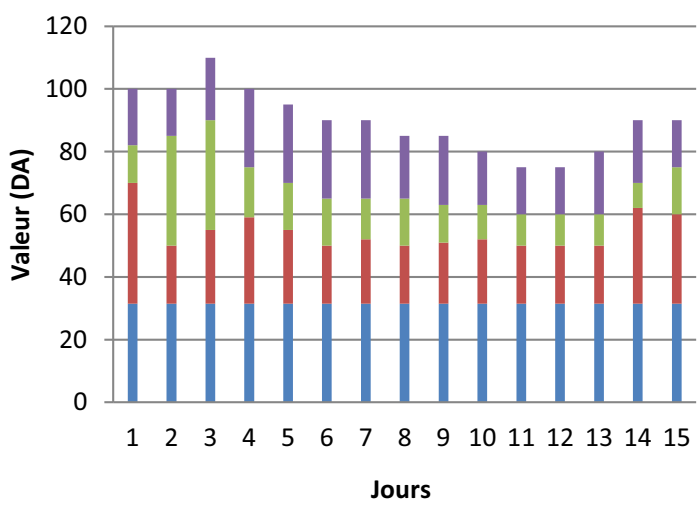

\author{
marge brute des détaillants \\ marge brute des grossistes \\ marge nette des producteurs \\ coûts de revient d'un $\mathrm{kg}$ de \\ tomate (Tofan) arrivé au \\ marché d'El Ghrous
}

Fig. 7. Variation des marges des intervenants $(\mathrm{DA} / \mathrm{kg})$.

Fig. 7. Added value variation ( $D A / \mathrm{kg})$.

formation des prix sur ce marché est principalement le résultat du jeu de l'offre et de la demande, affecté par des aléas exogènes et, à moins degré, endogènes.

Cela est en partie lié à la nature de la filière et du produit lui-même. La tomate primeur est un produit très périssable. Sa filière de commercialisation est simple, directe (sans stockage) et destinée au marché local (moyens de transport simples et sans taxe). Ces deux caractéristiques éliminent les possibilités de stockage et, de fait, diminuent les éventualités de spéculation. Sur d'autres produits maraîchers stockables, comme l'oignon ou la pomme de terre, les résultats seraient sans doute différents, car les temps de stockage laissent une marge plus grande aux pratiques spéculatives.

Ce marché est ainsi un atout majeur pour le développement de la plasticulture dans la région. Il contribue fortement à la dynamique locale de développement et d'extension de l'agriculture saharienne, caractérisée notamment par l'association des serres et des palmiers, la production sous serres étant elle-même porteuse du développement et de l'extension des palmeraies (Amichi et al., 2015). Les intermédiaires du marché sont ainsi des acteurs clés de ce développement, dans la mesure où non seulement ils assurent la transmission des informations relatives aux prix, mais aussi où ils relaient les exigences de la demande aux agriculteurs, en matière de qualité notamment. Comme dans d'autres pays du Maghreb, ces acteurs sont un des moteurs du développement de l'agriculture irriguée par les eaux souterraines (Lejars et al., à paraitre). Toutefois, la rentabilité de la filière étudiée, comme toutes les cultures pratiquées à Biskra, repose, d'une part, sur l'exploitation d'une ressource hydrique faiblement renouvelable et, d'autre part, sur l'utilisation intensive d'intrants et de produits phytosanitaires. Sur le long terme, la question de la disponibilité en eau et de la pollution par les produits phytosanitaires restent des défis majeurs pour la région comme pour les acteurs de ces filières. Une attention spéciale mériterait d'être accordée à ces aspects en s'intéressant particulièrement aux processus de transmission de la demande des consommateurs aux agriculteurs (pour des produits biologiques par exemple) et à la nature de la contribution des acteurs intermédiaires dans les processus de transmission d'informations.

\section{Références}

Amichi F, Bouarfa S, Lejars C, Kuper M, Hartani T, Daoudi A, et al. 2015. Des serres et des hommes : des exploitations motrices de l'expansion territoriale et de l'ascension socioprofessionnelle sur un front pionnier de l'agriculture saharienne en Algérie. Cahiers Agricultures 24: 11-19. DOI: 10.1684/agr.2015.0736.

Baci L. 1995. Les contraintes au développement du secteur des fruits et légumes en Algérie : faiblesse des rendements et opacité des marchés. Cahiers CIHEAM 14: 265-277. 
Boussard JM. 2010. Pourquoi l'instabilité est-elle une caractéristique structurelle des marches agricoles. Économie Rurale 320: $69-82$.

Daoudi A, Lejars C. 2016, De l'agriculture oasienne à l'agriculture saharienne dans la région des Ziban en Algérie. Acteurs du dynamisme et facteurs d'incertitude. New Medit 15(2): 45-52.

David-Benz H, Wade I, Egg J. 2005. Market information and price instability: an insight on vegetable markets in Senegal. In: ISHS Int. Conf., July 19-23, 2005, Chiang Mai.

Direction des services agricoles. 2013. Rapport: situation de l'agriculture de la wilaya de Biskra en 2013.

Galtier F. 2002. Information, institutions et efficacité des marchés - Trois filières céréalières d'Afrique de l'Ouest analysées comme des systèmes de communication. Thèse de doctorat en économie. Montpellier : Ensam. Disponible sur http://www.cormas.cirad.fr/ pdf/these FGaltier.pdf.

Galtier F, Bousquet F, Antona M, Bommel P. 2012. Markets as communication systems: Simulating and assessing the performance of market networks. Journal of Evolutionary Economics 22: 161-201.

Gouyon A. 1995. Compétitivité des filières et rôle des intermédiaires : Commercialisation de la production paysanne de caoutchouc naturel. Économie Rurale 228: 11-17.

Guillermou Y. 1994. Marchés, État et logiques paysannes en Algérie. Cahiers des sciences humaines 30: 111-133.
Lejars C, Courilleau S. 2015. Impact du développement de l'accès à l'eau souterraine sur la dynamique d'une filière irriguée. Le cas de l'oignon d'été dans le Saïs au Maroc. Cahiers Agricultures 24: 1-10. DOI: 10.1684/agr.2014.0729.

Lejars C, Daoudi A, Amichi H. 2017. The key role of supply chain actors in groundwater irrigation development in North Africa. Hydrogeology Journal. DOI: 10.1007/s10040-017- 1571-7.

Lothoré A, Delmas P. 2009. Accès au marché et commercialisation des produits agricoles : valorisation d'initiatives des producteurs. Édition Inter-réseaux, $176 \mathrm{p}$.

Ministère de l'Agriculture et du Développement rural. 2013. Rapport : situation de l'agriculture en Algérie en 2013.

Réflexion. 10 mars 2015. Disponible sur http://www.reflexiondz.net/ MASCARA-La-pomme-de-terre-victime-des-speculateursa33367.html, article de presse.

Sah̆li Z. 2009. Produits de terroir et développement local en Algérie: cas des zones rurales de montagnes et de piémonts. Cahiers CIHEAM 89: 305-338.

Tarchiani V, Robbiati G, Salifou MR. 2013. Filières oignon en Afrique de l'Ouest : étude comparée des filières nigérienne et béninoise. Cahiers Agricultures 22: 112-123. DOI: 10.1684/agr.2013.0617.

Zoubeidi M, Chehat F. 2011. Le fonctionnement du marché des ovins dans les hautes plaines steppiques de l'Ouest Algérien : entre contraintes et répartition de la valeur. Livestock Research for Rural Development 23: 9 .

Citation de l'article : Assassi S, Daoudi A, Lejars C. 2017. Les profits « excessifs » des commerçants de fruits et légumes en Algérie : réalité ou préjugé infondé ? Le cas de la tomate primeur à Biskra. Cah. Agric. 26: 25002. 